\title{
Non-parallelism between Reaction Rate and Dienophile-Catalyst Affinity in Catalytic Enantioselective Diels-Alder Reactions
}

\author{
Do Hyun Ryu, Gang Zhou and E. J. Corey* \\ Department of Chemistry and Chemical Biology \\ Harvard University, Cambridge, Massachusetts, 02138, USA \\ Department of Chemistry, Sungkyunkwan University, Suwon 440-746, Korea
}

\section{$\underline{\text { Supplementary Materials }}$}

Materials and Methods. Thin-layer chromatography (TLC) was performed using E. Merck silica gel $60 \mathrm{~F}_{254}$ precoated plates $(0.25 \mathrm{~mm})$. Flash chromatography was performed using Baker silica gel (40 $\mu \mathrm{m}$ particle size). NMR spectra were recorded on Varian Innova-500 or Mercury400 instruments and calibrated using residual undeuterated solvent as an internal reference. IR spectra were recorded on Avatar 360 FT-IR spectrometer. Low-resolution mass (CI) spectra were obtained by using a Platform II mass spectrometer. Analytical high performance liquid chromatography (HPLC) was performed on Agilent 1100 Series HPLC using the indicated chiral column. Gas chromatography (GC) analyses were performed on Hewlett-Packard 6850 Series GC System equipped with flame ionization detector using a J \& W Scientific Cyclosil-B (30 m x $0.25 \mathrm{~mm})$ column or an Alltech Chiraldex $\gamma$-TA $(30 \mathrm{~m} \times 0.25 \mathrm{~mm})$ column. Dichloromethane, methyl acrylate and methacrolein were distilled from calcium hydride.

Illustrative Procedure for a Typical Competition Experiment. The solution of oxazaborolidinium catalyst $(0.1 \mathrm{mmol})$ in dichloromethane $(7.5 \mathrm{ml})$ was prepared from $(S)-(-)-$ a, $\alpha$-diphenyl-2-pyrrolidinemethanol (30.4 mg, $0.12 \mathrm{mmol})$, tri-o-tolylboroxine (14.2 $\mathrm{mg}, 0.04$ $\mathrm{mmol})$ and triflimide $\left(0.5 \mathrm{ml}, 0.2 \mathrm{M}\right.$ in dichloromethane) by the previously reported procedure. ${ }^{1}$ To a stirred solution of trifluoroethyl acrylate $(127 \mu 1,1 \mathrm{mmol})$ and methyl acrylate $(68.6 \mu 1,1$ $\mathrm{mmol})$ in dichloromethane $(1 \mathrm{ml})$ was added the freshly prepared catalyst solution $(0.05 \mathrm{mmol}, 4$

\footnotetext{
${ }^{1}$ Ryu, D. H.; Corey, E. J. J. Am. Chem. Soc. 2003, 125, 6388-6390.
} 
ml) at $-78^{\circ} \mathrm{C}$. Half of the catalyst solution was used for the control experiment without methyl acrylate. After $10 \mathrm{~min}, 1,3$-cyclopentadiene $(248 \mu \mathrm{l}, 3 \mathrm{mmol})$ was added at $-78^{\circ} \mathrm{C}$. An aliquot $(50 \mu \mathrm{l})$ was removed from the reaction mixture and quenched quickly with $\mathrm{Et}_{3} \mathrm{~N}(20 \mu \mathrm{l})$ solution in $\mathrm{CDCl}_{3}(0.6 \mathrm{ml})$. The progress of the reaction as a function of time was determined by ${ }^{1} \mathrm{H} \mathrm{NMR}$ integration (500 MHz, $\mathrm{CDCl}_{3}$ ): $\delta 4.54$ (q, 2H, $J=8.5 \mathrm{~Hz}$, trifluoroethyl acrylate), 4.35 (dq, 1H, $J$ $=12.8,8.4 \mathrm{~Hz}$, adduct of trifluoroethyl acrylate), $3.75(\mathrm{~s}, 3 \mathrm{H}$, methyl acrylate), $3.62(\mathrm{~s}, 3 \mathrm{H}$, adduct of methyl acrylate).

\section{Determination of Enantioselectivity of the Diels-Alder Adducts.}

Compound 11: Enantioselectivity was determined by desilylation with $10 \%$ HF to the corresponding ketone and $\mathrm{GC}$ analysis (Cyclosil-B, $155{ }^{\circ} \mathrm{C}, 25 \mathrm{psi}$ ); retention times: 58.4 (major), $59.7 \mathrm{~min}$ (minor).

Compound 13 ( $\left.\mathbf{Y}=\mathbf{C F}_{3} \mathbf{C H}_{2} \mathbf{O}\right)$ : Enantioselectivity was determined by GC analysis $(\gamma-\mathrm{TA}, 100$ ${ }^{\circ} \mathrm{C}, 22 \mathrm{psi}$ ); retention times: 6.4 (endo, major), $7.1 \mathrm{~min}$ (endo, minor).

Compound 13 ( $\mathbf{Y = C l ) : ~ E n a n t i o s e l e c t i v i t y ~ w a s ~ d e t e r m i n e d ~ b y ~ c o n v e r s i o n ~ t o ~ t h e ~ m e t h y l ~ e s t e r ~}$ $\left(\mathrm{Y}=\mathrm{CH}_{3} \mathrm{O}\right)$ and $\mathrm{GC}$ analysis $\left(\gamma-\mathrm{TA}, 100{ }^{\circ} \mathrm{C}, 22 \mathrm{psi}\right)$; retention times: 6.4 (endo, major), 6.6 min (endo, minor).

Compound 13 (Y= OH): Enantioselectivity was determined by transformation ${ }^{2}$ with oxalyl chloride to the acid chloride, conversion to the ethyl ester $\left(\mathrm{Y}=\mathrm{CH}_{3} \mathrm{CH}_{2} \mathrm{O}\right)$ and $\mathrm{GC}$ analysis (Cyclosil-B, $95^{\circ} \mathrm{C}, 25 \mathrm{psi}$ ); retention times: 15.4 (endo, major), $16.1 \mathrm{~min}$ (endo, minor).

Compound 14: Enantioselectivity was determined by GC analysis (Cyclosil-B, $90{ }^{\circ} \mathrm{C}, 25 \mathrm{psi}$ ); retention times: $77.0 \mathrm{~min}$ (endo, minor), $77.9 \min$ (endo, major).

\footnotetext{
${ }^{2}$ Stille, J. R.; Santarsiero, B. D.; Grubbs, R. H. J. Org. Chem. 1990, 55, 843.
} 
Compound 15 and 16: Enantioselectivity was determined by transformation ${ }^{2}$ with oxalyl chloride to the acid chloride, conversion to the diethyl ester and GC analysis (Cyclosil-B, 110 ${ }^{\circ} \mathrm{C}, 25 \mathrm{psi}$ ); retention times: 68.3 (minor), 69.9 min (major).

Physical Data of the Diels-Alder Products and Experimental Procedure:

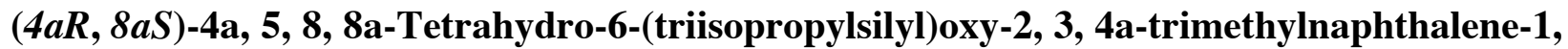
4-dione (11). The physical and spectral data were identical to those previously reported for this compound ${ }^{3}:{ }^{1} \mathrm{H}$ NMR $\left(400 \mathrm{MHz}, \mathrm{CDCl}_{3}\right) \delta 4.80(\mathrm{t}, 1 \mathrm{H}, J=3.6 \mathrm{~Hz}), 2.74(\mathrm{dd}, 1 \mathrm{H}, J=6.0$, $5.2 \mathrm{~Hz}), 2.56-2.68(\mathrm{~m}, 1 \mathrm{H}), 2.52(\mathrm{dd}, 1 \mathrm{H}, J=17.2,1.6 \mathrm{~Hz}), 2.12-2.22(\mathrm{~m}, 1 \mathrm{H}), 1.99(\mathrm{~s}, 3 \mathrm{H}), 1.96$ (s, 3H), $1.79(\mathrm{~d}, 1 \mathrm{H}, J=17.2 \mathrm{~Hz}), 1.32(\mathrm{~s}, 3 \mathrm{H}), 1.01-1.12(\mathrm{~m}, 3 \mathrm{H}), 1.03(\mathrm{~d}, 18 \mathrm{H}, J=6.0 \mathrm{~Hz}) ;{ }^{13} \mathrm{C}$ NMR (100 MHz, $\left.\mathrm{CDCl}_{3}\right) \delta$ 201.9, 199.6, 147.8, 143.6, 142.6, 99.9, 51.8, 48.8, 37.1, 23.6, 23.1, $18.2(x 6), 13.4,13.1,12.8(x 3)$

$(1 R, 2 R, 4 R)$-Bicyclo[2.2.1]hept-5-ene-2-carboxylic acid 2,2,2-trifluoro-ethyl ester (13, Y= $\mathrm{CF}_{3} \mathrm{CH}_{2} \mathbf{O}$ ). The physical and spectral data were identical to those previously reported for this compound ${ }^{4}:{ }^{1} \mathrm{H} \mathrm{NMR}\left(400 \mathrm{MHz}, \mathrm{CDCl}_{3}\right) \delta 6.22(\mathrm{dd}, 1 \mathrm{H}, J=5.6,3.2 \mathrm{~Hz}), 5.92(\mathrm{dd}, 1 \mathrm{H}, J=5.6$, $2.8 \mathrm{~Hz}), 4.49(\mathrm{dq}, 1 \mathrm{H}, J=12.8,8.4 \mathrm{~Hz}), 4.35(\mathrm{dq}, 1 \mathrm{H}, J=12.8,8.4 \mathrm{~Hz}), 3.26(\mathrm{~s}, 1 \mathrm{H}), 3.04(\mathrm{dt}$, $1 \mathrm{H}, J=4.0,9.2 \mathrm{~Hz}), 2.94(\mathrm{~s}, 1 \mathrm{H}), 1.95(\mathrm{ddd}, 1 \mathrm{H}, J=11.6,9.2,3.6 \mathrm{~Hz}), 1.42-1.48(\mathrm{~m}, 2 \mathrm{H}), 1.30$ $(\mathrm{d}, 1 \mathrm{H}, J=8.0 \mathrm{~Hz}) ;{ }^{13} \mathrm{C} \mathrm{NMR}\left(100 \mathrm{MHz}, \mathrm{CDCl}_{3}\right) \delta 173.1,138.1,132.0,123.0$ (q, $1 \mathrm{C}, J=276$ $\mathrm{Hz}), 60.0(\mathrm{q}, 1 \mathrm{C}, J=36.4 \mathrm{~Hz}), 49.6,45.8,42.9,42.5,29.2$.

$(1 R, 2 R, 4 R)$-Bicyclo[2.2.1] hept-5-ene-2-carboxylic acid methyl ester $\left(13, \mathrm{Y}=\mathrm{CH}_{3} \mathrm{O}\right)$. The physical and spectral data were identical to those previously reported for this compound ${ }^{5}:{ }^{1} \mathrm{H}$

\footnotetext{
${ }^{3}$ Ryu, D. H.; Zhou, G.; Corey, E. J. J. Am. Chem. Soc. 2004, 126, 4800-4802.

${ }^{4}$ Ryu, D. H.; Corey, E. J. J. Am. Chem. Soc. 2003, 125, 6388-6390.

5 a) Hawkins, J. M.; Loren, S. J. Am. Chem. Soc. 1991, 113, 7794. b) Hawkins, J. M.; Loren, S.; Nambu, M. J. Am. Chem. Soc. 1994, 116, 1657.
} 
NMR (400 MHz, $\left.\mathrm{CDCl}_{3}\right) \delta 6.20(\mathrm{dd}, 1 \mathrm{H}, J=5.6,3.2 \mathrm{~Hz}), 5.92(\mathrm{dd}, 1 \mathrm{H}, J=5.6,3.2 \mathrm{~Hz}), 3.62(\mathrm{~s}$, 3H), 3.20(s, 1H), $2.94(\mathrm{dt}, 1 \mathrm{H}, J=9.2,4.0 \mathrm{~Hz}), 2.90(\mathrm{~s}, 1 \mathrm{H}), 1.90(\mathrm{ddd}, 1 \mathrm{H}, J=11.6,9.2,3.6$ $\mathrm{Hz}), 1.36-1.43(\mathrm{~m}, 2 \mathrm{H}), 1.27(\mathrm{~d}, 1 \mathrm{H}, J=8.4 \mathrm{~Hz})$.

$(1 R, 2 R, 4 R)$-Bicyclo[2.2.1]hept-5-ene-2-carboxylic acid (13, Y= OH). The physical and spectral data were identical to those previously reported for this compound ${ }^{6}:{ }^{1} \mathrm{H}$ NMR $(500 \mathrm{MHz}$, $\left.\mathrm{CDCl}_{3}\right) \delta 11.76(\mathrm{brs}, 1 \mathrm{H}), 6.21(\mathrm{dd}, 1 \mathrm{H}, J=3.0,5.0 \mathrm{~Hz}), 6.01(\mathrm{dd}, 1 \mathrm{H}, J=5.0,3.0 \mathrm{~Hz}), 3.24(\mathrm{~s}$, 1H), $2.98(\mathrm{dt}, 1 \mathrm{H}, J=9.5,4.0 \mathrm{~Hz}), 2.92(\mathrm{~s}, 1 \mathrm{H}), 1.90-1.95(\mathrm{~m}, 1 \mathrm{H}), 1.44-1.47(\mathrm{~m}, 1 \mathrm{H}), 1.39-1.43$ $(\mathrm{m}, 1 \mathrm{H}), 1.29(\mathrm{~d}, 1 \mathrm{H}, J=8.0 \mathrm{~Hz})$.

$(1 R, 2 S, 3 S, 4 S)$ - bis(2,2,2-trifluoroethyl) bicyclo[2.2.1]hept-5-ene-2,3-dicarboxylate (14). ${ }^{1} \mathrm{H}$ NMR (400 MHz, $\left.\mathrm{CDCl}_{3}\right) \delta$ 6.33(dd, $\left.1 \mathrm{H}, J=3.6,5.7 \mathrm{~Hz}\right), 6.08(\mathrm{dd}, 1 \mathrm{H}, J=3.6,5.7 \mathrm{~Hz}), 4.32-$ $4.61(\mathrm{~m}, 4 \mathrm{H}), 3.49(\mathrm{t}, 1 \mathrm{H}, J=3.9 \mathrm{~Hz}), 3.36(\mathrm{bs}, 1 \mathrm{H}), 3.20(\mathrm{bs}, 1 \mathrm{H}), 2.80(\mathrm{dd}, 1 \mathrm{H}, J=1.8,4.5 \mathrm{~Hz})$, $1.62(\mathrm{~d}, 1 \mathrm{H}, J=9.0 \mathrm{~Hz}), 1.52(\mathrm{~d}, 1 \mathrm{H}, J=9.0 \mathrm{~Hz}) ;{ }^{13} \mathrm{C} \mathrm{NMR}\left(100 \mathrm{MHz}, \mathrm{CDCl}_{3}\right) \delta 172.8,171.5$, 138.0, 135.2, $123.5(\mathrm{q}, 2 \mathrm{C}, J=275 \mathrm{~Hz}), 60.7$ (2C), 47.9, 47.8, 47.5, 47.1, 46.0; FTIR (neat) $1746,1277,1147,1110,976 ;[\alpha]_{\mathrm{D}}^{23}+132\left(c 1.1, \mathrm{CHCl}_{3}, 95 \%\right.$ \%e $)$.

$(1 R, 2 S, 3 S, 4 S)$-Bicyclo[2.2.1]hept-5-ene-2,3-dicarboxylic acid, mono-3-ethyl ester (15) and $(1 R, 2 S, 3 S, 4 S)$-Bicyclo[2.2.1]hept-5-ene-2,3-dicarboxylic acid, mono-2-ethyl ester (16).

The catalytic Diels-Alder reaction with monoethyl fumarate $(0.667 \mathrm{mmol})$ by the previously reported procedure ${ }^{1,4 \mathrm{c}}$ provided $137 \mathrm{mg}(99 \%)$ of a mixture of $\mathbf{1 5}$ and $\mathbf{1 6}\left(82: 18\right.$ by ${ }^{1} \mathrm{H}$ NMR integration) as colorless oil after silica gel column chromatography (gradient elution with 10$60 \%$ EtOAc-hexanes).

\footnotetext{
${ }^{6}$ (a) Sarakinos, G.; Corey, E. J. Org. Lett. 1999, 1, 1741. (b) Poll, T.; Sobczak, A.; Hartmann, H.; Helmchen, G. Tetrahedron . Lett. 1985, 26, 3095. (c) Ryu, D. H.;Lee, T..W.; Corey, E. J. J. Am. Chem. Soc. 2002, 124, 99929993.
} 
Iodolactonization; To a stirred solution of acids $\mathbf{1 5}$ and $\mathbf{1 6}$ (82:18 ration, 137mg, $0.65 \mathrm{mmol})$ in dichloromethane $(1 \mathrm{~mL})$ were added sodium hydrogen carbonate $(62 \mathrm{mg}, 0.74 \mathrm{mmol})$, water $(3$ $\mathrm{mL}$ ), potassium iodide (398 $\mathrm{mg}, 2.4 \mathrm{mmol}$ ) and iodine (203 $\mathrm{mg}, 0.80 \mathrm{mmol})$. The mixture was stirred for $1 \mathrm{~h}$ then saturated sodium thiosulfate solution $(1.7 \mathrm{~mL})$ was added. The aqueous layer was extracted with EtOAc $(4 \mathrm{~mL} \times 3)$. The combined extract was dried over sodium sulfate and concentrated in vacuo to give oily residue. Column chromatography (gradient elution with 10$60 \%$ EtOAc-hexanes) afforded $175 \mathrm{mg}(80 \%)$ of iodolactone as a colorless oil and $25 \mathrm{mg}(18 \%)$ of unreacted minor adduct $\mathbf{1 6}$ as a colorless oil. The physical and spectral data were identical to those previously reported for these compounds ${ }^{7}$.

Iodolactone; ${ }^{1} \mathrm{H}$ NMR $\left(500 \mathrm{MHz}, \mathrm{CDCl}_{3}\right) \delta 5.12(\mathrm{~d}, 1 \mathrm{H}, J=5.0 \mathrm{~Hz}), 4.14-4.19(\mathrm{~m}, 2 \mathrm{H}), 3.88(\mathrm{~d}$, $1 \mathrm{H}, J=2.5 \mathrm{~Hz}), 3.19-3.21(\mathrm{~m}, 1 \mathrm{H}), 3.09(\mathrm{brd}, 1 \mathrm{H}, J=5.0 \mathrm{~Hz}), 3.00$ (brs, 1H), 2.81 (brs, 1H), $2.30(\mathrm{dd}, 1 \mathrm{H}, J=1.5,12.5 \mathrm{~Hz}), 1.93-1.96(\mathrm{~m}, 1 \mathrm{H}), 1.26(\mathrm{t}, 3 \mathrm{H}, J=7.0 \mathrm{~Hz}) ;[\alpha]_{\mathrm{D}}^{23}-40.0(c 1.0$, $\mathrm{CHCl}_{3}, 97 \%$ ee).

$16 ;{ }^{1} \mathrm{H} \mathrm{NMR}\left(500 \mathrm{MHz}, \mathrm{CDCl}_{3}\right) \delta 6.29(\mathrm{dd}, 1 \mathrm{H}, J=4.0,7.0 \mathrm{~Hz}), 6.08(\mathrm{dd}, 1 \mathrm{H}, J=3.0,7.0 \mathrm{~Hz})$, 4.07-4.13 (m, 2H), $3.36(\mathrm{t}, 1 \mathrm{H}, J=5.0 \mathrm{~Hz}), 3.28(\mathrm{brs}, 1 \mathrm{H}), 3.20(\mathrm{~m}, 1 \mathrm{H}), 2.74(\mathrm{dd}, 1 \mathrm{H}, J=1.5$, $4.5 \mathrm{~Hz}), 1.61(\mathrm{brd}, 1 \mathrm{H}, J=11.0 \mathrm{~Hz}), 1.47-1.50(\mathrm{~m}, 1 \mathrm{H}), 1.24(\mathrm{t}, 3 \mathrm{H}, J=8.5 \mathrm{~Hz}) ;[\alpha]_{\mathrm{D}}^{23}+130(c$ 1.0, $\mathrm{CHCl}_{3}, 98 \%$ ee).

Zinc Reduction; To a stirred solution of iodolactone (152 $\mathrm{mg}, 0.452 \mathrm{mmol})$ in acetic acid (1.5 $\mathrm{mL}$ ) was added zinc (powder, 148mg, $2.26 \mathrm{mmol}$ ) and the reaction mixture was stirred at $65{ }^{\circ} \mathrm{C}$ for $1 \mathrm{hr}$. Then water $(8 \mathrm{~mL})$ was added and the mixture was extracted with ethyl acetate $(5 \mathrm{~mL} \mathrm{x}$ 3). The combined extract was dried over anhydrous sodium sulfate and concentrated to give the crude product. This crude product was purified by silica gel chromatography (gradient elution with 10-60\% EtOAc-hexanes) to furnish $94 \mathrm{mg}(99 \%)$ of $\mathbf{1 5}$ as a colorless oil. The physical and spectral data were identical to those previously reported for this compound ${ }^{7}:{ }^{1} \mathrm{H}$ NMR $(500 \mathrm{MHz}$, $\left.\mathrm{CDCl}_{3}\right) \delta 6.27(\mathrm{dd}, 1 \mathrm{H}, J=3.5,5.5 \mathrm{~Hz}), 6.08(\mathrm{dd}, 1 \mathrm{H}, J=2.5,5.5 \mathrm{~Hz}), 4.15(\mathrm{q}, 2 \mathrm{H}, J=7.0 \mathrm{~Hz})$,

\footnotetext{
${ }^{7}$ Clapham G.; Shipman, M. Tetrahedron 2000, 56, 1127-1134.
} 
$3.42(\mathrm{t}, 1 \mathrm{H}, J=4.0 \mathrm{~Hz}), 3.27(\mathrm{brs}, 1 \mathrm{H}), 3.11(\mathrm{~m}, 1 \mathrm{H}), 2.61(\mathrm{dd}, 1 \mathrm{H}, J=1.5,4.5 \mathrm{~Hz}), 1.60$ (brd, $1 \mathrm{H}, J=9.0 \mathrm{~Hz}), 1.44(\mathrm{dd}, 1 \mathrm{H}, J=1.5,9.0 \mathrm{~Hz}), 1.25(\mathrm{t}, 3 \mathrm{H}, J=7.5 \mathrm{~Hz}) ;[\alpha]_{\mathrm{D}}^{23}+136(c 1.0$, $\mathrm{CHCl}_{3}, 97 \%$ ee). 